COMMENT. Acquired cerebellar lesions have been associated with a 'cerebellar cognitive affective syndrome, [CCAS].' (Schmahmann JD, Sherman JC. Brain 1998;121:561-579). CCAS is characterized by disorders of executive cognitive functioning, visuospatial ability, expressive language, working memory, and affective behavior. Patients have impairment of planning, abstract reasoning, verbal fluency, working memory, impaired spatial cognition associated with distractibility, perseveration, and inattention, anomia, and personality change with blunting of affect or disinhibited or inappropriate behavior. Vermal lesions are associated with marked affective and communication disorders, or with postsurgical mutism and speech or language disorders. CCAS is described in both adults and in children with acquired cerebellar lesions. The above study has shown that CCAS also occurs with cerebellar malformations. It is noteworthy that $9(33 \%)$ patients were diagnosed with PDD and 2 with ADHD. A structural brain pathology as the basis for ADHD is also supported by an NIH MRI study showing a smaller cerebellar vermis in boys with ADHD, particularly involving posterior inferior lobules VIII to X. (Berquin PC et al. Neurology 1998;50:1087-1093). The cerebellum controls not only motor functions but also cognitive, language, behavioral and affective functions.

\title{
NEUROFIBROMATOSIS 1, THALAMIC HYPERINTENSITIES, AND COGNITIVE IMPAIRMENT
}

The relationship between T2 weighted MRI images (T2H) and cognitive functioning in a cohort of 76 children with neurofibromatosis type 1 (NF1) was determined in a study at Children's Hospital at Westmead, University of Sydney, Australia. Patients ranged in age from 8.0 to 16.75 (mean 11.63). NF1 was sporadic in $61 \%$ and familial in $39 \%$. One-half were macrocephalic. Ninety percent had $\mathrm{T} 2 \mathrm{H}$, and $71 \%$ had discrete $\mathrm{T} 2 \mathrm{H}$ lesions. Those with $\mathrm{T} 2 \mathrm{H}$ lesions were slightly younger than those without ( $11.4 \mathrm{vs} 13.3 \mathrm{yrs} ; \mathrm{p}=0.029$ ), and a greater number of $\mathrm{T} 2 \mathrm{H}$ were found in younger children. Discrete lesions were usually located in the basal ganglia and cerebral hemispheres, whereas diffuse lesions involved the thalamus, cerebellum and brainstem. T2 $\mathrm{H}$ in general were not associated with cognitive dysfunction, specific learning disabilities or ADHD, but patients with discrete thalamic lesions showed lower levels of cognitive functioning than those without lesions or with lesions elsewhere (Full scale IQ 72.8 vs 91.4). Thalamic lesions were also associated with lower performance on Verbal IQ, Performance IQ, Processing Speed Index, spelling, sustained attention, fine motor coordination, and motor speed. More diffuse lesions in the thalamus were also associated with reductions in IQ but less marked than those with discrete lesions. (Hyman SL, Gill DS, Shores EA, Steinberg A, North KN. T2 hyperintensities in children with neurofibromatosis type 1 and their relationship to cognitive functioning. J Neurol Neurosurg Psychiatry October 2007;78:1088-1091) (Respond: Dr Kathryn North, Clinical School, the Children's Hospital at Westmead, Locked Bag 4001, Westmead NSW 2145, Australia).

COMMENT. Thalamic lesions, particularly when discrete, are associated with cognitive impairment in children with NF1. Previous studies have shown that high-signal MRI lesions in NFl evolve over time. They either increase or decrease in size or number, dependent on their location. Lesions in the thalamus, brainstem and cerebellar peduncles expand whereas those in cerebral hemispheres and cerebellum regress. Surgery or 
radiotherapy was required for mass lesions in 3 patients followed for a mean time of 2 to 3 years. (DiMario FJ Jr, Ramsby G. Arch Neurol 1998;55:500-505).

\section{PREFRONTAL CORTICAL ASYMMETRY AND ATTENTION DEFICIT HYPERACTIVITY DISORDER}

Fractal information dimension (FID), a scale-free measure, was used to assess the prefrontal cortical convolution complexity and asymmetry in 12 boys with ADHD and 11 controls, in an MRI study at the Chinese Academy of Sciences, and other centers in Beijing, PR China. A left-greater-than-right prefrontal cortical convolution complexity was present in both groups, but in ADHD patients the complexity pattern was significantly reduced. This resulted in a significant reduction of the normal prefrontal cortical asymmetry pattern in ADHD compared to control subjects. (Li X, Jiang J, Zhu W et al. Asymmetry of prefrontal cortical convolution complexity in males with attention-deficit/hyperactivity disorder using fractal information dimension. Brain Dev Nov 2007;29:649-655). (Respond: E-mail: jiangtz(onlpr.ia.ac.cn (T Jiang)).

COMMENT. MRI volumetric studies have shown prefrontal cortical abnormalities in ADHD, sometimes affecting the right prefrontal and other brain regions. (Castellanos FX et al. Arch Gen Psychiatry 1996;53:607-616). The current study assesses the cortical convolution (or gyrification) complexity by using the fractal information dimension, a scalefree measure unaffected by variations in volume of the brain region. A greater FID value shows a higher cortical convolution level of cerebral sulci and gyri. The study found a significantly reduced cortical convolution complexity in the left prefrontal region in ADHD boys. Other studies have found volume reduction in the left prefrontal cortex in children and adults with ADHD, and reduced left prefrontal cortical thickness and cerebral blood flow in ADHD children. The findings indicate an underdevelopment of the left prefrontal lobe in ADHD, in support of an organic neurological basis for the syndrome. A frontal-motor cortex disconnection syndrome, or 'lazy' frontal lobe, in ADHD is one hypothesis, resulting in failure of the frontal lobe to inhibit excessive motor activity and sustain attention. (Niedermeyer E, Naidu SB. Clin Electroencephalogr 1997;28:130-136). The frontal lobe stimulant effect of methylphenidate corrects the impaired motor inhibition.

Acute dyskinesia on starting methylphenidate after risperidone withdrawal is reported in a 7-year-old boy with conduct disorder and ADHD (Hollis CP et al. Pediatr Neurol Oct 2007;37:287-288). Within 8 hours of taking Concerta $36 \mathrm{mg}$, the boy manifested twitching of hands and feet, throat clearing, overactivity, and vomiting. After risperidone reinstatement $1.5 \mathrm{mg}$, tics resolved within a few hours. A second attempt to substitute Concerta $18 \mathrm{mg}$ for risperidone, 5 months later, resulted in almost immediate mouthing movements, tongue protrusion, shoulder shrugging, and dystonic flexion of wrist and fingers. Movements resolved by the following morning, and a trial of atomoxetine provided adequate control of ADHD and aggressive behavior. The authors advise caution in the use of combinations of risperidone and psychostimulants. Slow neuroleptic withdrawal and extended washout period should precede initiation of stimulant medication in low dose. 